Check for updates

Cite this: J. Mater. Chem. C, 2020 8, 15199

Received 11th June 2020,

Accepted 27th July 2020

DOI: $10.1039 / \mathrm{dOtc02774j}$

rsc.li/materials-c

\section{Fermi level pinned molecular donor/acceptor junctions: reduction of induced carrier density by interfacial charge transfer complexes $\dagger$}

\author{
Paul Beyer, ${ }^{a}$ Eduard Meister, ${ }^{\mathrm{b}}$ Timo Florian, $^{\mathrm{a}}$ Alexander Generalov, ${ }^{\mathrm{c}}$ \\ Wolfgang Brütting, (D) ${ }^{b}$ Norbert Koch (D)*ad and Andreas Opitz (D) *a
}

\begin{abstract}
Increased hole density in an electron donor-type organic semiconductor can be achieved by deposition of a strong acceptor-type molecular layer on top, and has been shown to enable adjusting the carrier density in organic field effect transistors (OFETs). This interfacial charge transfer is due to simultaneous Fermi level $\left(E_{\mathrm{F}}\right)$ pinning of the donor's highest occupied level and the acceptor's lowest unoccupied level. Here, we investigate the electrical properties of such an $E_{\mathrm{F}}$-pinned junction formed by diindenoperylene (DIP, as donor) and hexafluoro-tetracyano-naphthoquinodimethane (F6, as acceptor) in OFETs, as well as its electronic properties by photoelectron spectroscopy and electrostatic modelling. We find that, in addition to the $E_{\mathrm{F}}$-pinning induced integer charge transfer across the interface, DIP and F6 form charge transfer complexes (CPXs) at their junction. The molecularly thin CPX interlayer acts as insulator and significantly reduces the density of carriers induced on either side of the junction, compared to a scenario without such an interlayer. CPX formation is thus unfavourable for the effectiveness of controlling carrier density at molecular donor/acceptor junctions by $E_{\mathrm{F}}$-pinning.
\end{abstract}

\section{Introduction}

The interest in combinations of electron donor (D) and acceptor (A) molecular materials has significantly grown in the recent past. Prototypical D-A combinations are used in photovoltaic cells, ${ }^{1}$ exciplex emitters, ${ }^{2}$ and doped charge transport layers, ${ }^{3}$ where the occurrence of ground- or excited-state charge transfer (CT) depends strongly on the energy levels of the involved compounds. For ground-state CT, one should recall that either an integer charge can be transferred between $\mathrm{D}$ and $\mathrm{A}$ or charge transfer complex (CPX) formation can prevail. ${ }^{4}$ The latter requires significant overlap and hybridization of the $\mathrm{D}$ highest occupied molecular orbital (HOMO) and the A lowest unoccupied molecular orbital (LUMO), resulting in a new set of CPX frontier energy levels. Therefore, the CPX exhibits a lower energy gap compared to its parent compounds, and often D-A co-crystals are formed upon mixing $\mathrm{D}$ and $\mathrm{A}$. These have been under

\footnotetext{
${ }^{a}$ Institut für Physik \& IRIS Adlershof, Humboldt-Universität zu Berlin, Germany. E-mail:norbert.koch@physik.hu-berlin.de, andreas.opitz@hu-berlin.de

${ }^{b}$ Institute of Physics, University of Augsburg, Germany

${ }^{c}$ Max IV Laboratory, Lund University, Sweden

${ }^{d}$ Helmholtz-Zentrum Berlin für Materialien und Energie GmbH, Germany

$\dagger$ Electronic supplementary information (ESI) available: Source-gate current measurements, additional structural data from X-ray reflectivity and scanning force microscopy, absorption spectra, angle dependent NEXAFS deconvolution, parameters used for electrostatic modelling. See DOI: 10.1039/d0tc02774j
}

investigation as narrow gap semiconductors, ${ }^{5}$ in infrared detectors, ${ }^{6}$ and the CPX as dopant in organic semiconductor matrices. ${ }^{7}$

Planar two-layer structures of separate D and A on top of each other have been investigated in organic field-effect transistors (OFET) ${ }^{8-11}$ as this provides for transport channels for both electrons and holes in the two semiconductor layers. It was observed that the threshold voltage in two-layer OFETs differs from the respective single-layer devices, which was related to CT and thus local doping at the D-A interface. ${ }^{8-11} \mathrm{An}$ increased charge carrier density, associated with (partial) filling of trap states can indeed result in a lower threshold voltage. ${ }^{12,13}$ Electrostatic modelling has highlighted that integer CT at D-A heterojunction interfaces can occur if needed to reach electronic equilibrium, with respective charge accumulation and energy level bending on either side of the junction. ${ }^{14}$ This type of CT can be long-range and has been described within the framework of Fermi level $\left(E_{\mathrm{F}}\right)$ pinning, ${ }^{14,15}$ originally developed for electrode-semiconductor contacts. ${ }^{16,17}$ This phenomenon has enabled the observation of metallic conductivity at a junction between two molecular D and A single crystals, ${ }^{18}$ and, more recently, substantial ground-state $\mathrm{CT}$ at conjugated polymer heterojunctions. ${ }^{19}$

However, the analysis of molecular D-A systems is challenging because of the crucial interplay between morphology, energy level alignment, and thus $\mathrm{CT}^{20}{ }^{20}$ Junctions of dissimilar molecules can 
(a)

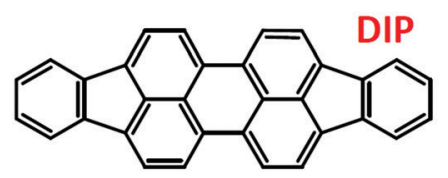

(b)

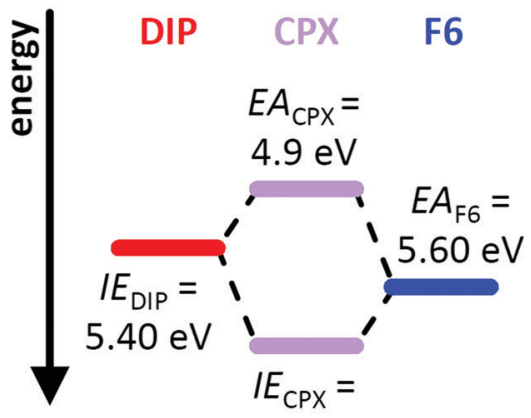

(c)

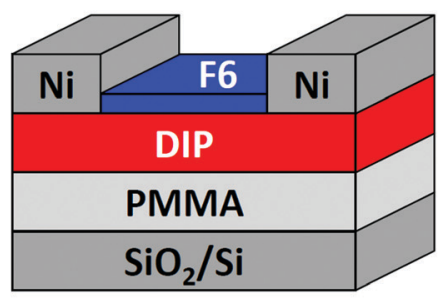

$6.1 \mathrm{eV}$

Fig. 1 (a) Chemical structure of the organic semiconductor diindenoperylene (DIP) and the acceptor hexafluoro-tetracyano-naphthoquinodimethane (F6). (b) Schematic energy level diagram of DIP, F6 and the formed charge-transfer complex (CPX). Ionisation energy (IE) of DIP and electron affinity (EA) of F6 are taken from literature and were determined by direct and inverse photoelectron spectroscopy. ${ }^{32,33,36,37}$ (c) Sketch of the device structure for DIP field-effect transistors. The devices were characterised before and after deposition of F6 molecules.

be fairly sharp and stable, ${ }^{21}$ while others can undergo local re-orientation ${ }^{22}$ of molecules or intermixing upon interface formation. ${ }^{23,24}$ These structural details significantly impact the nature of the CT, and the presence or absence of D-A $\pi$-stacking can significantly modify the optical properties of the heterojunction. ${ }^{25,26}$ Therefore, a comprehensive understanding of D-A heterojunctions is required to relate the fundamental properties to the performance of pertinent devices, and for their improvement.

In this contribution, we demonstrate how two - simultaneously occurring - types of CT (CPX formation and $E_{\mathrm{F}}$-pinning) at a molecular D-A junction modify the charge carrier density distribution across the two layers, and how this impacts the electrical characteristics of corresponding OFETs. This is exemplified for diindenoperylene $^{27,28}$ (DIP) as donor and hexafluoro-tetracyanonaphthoquinodimethane (F6) as strong electron acceptor; ${ }^{29-31}$ the chemical structure of both compounds are displayed in Fig. 1a. The reported electron affinity (EA) of $\mathrm{F} 6$ thin films $\left(\mathrm{EA}_{\mathrm{F} 6}=\right.$ $5.60 \mathrm{eV})^{32}$ is higher than the ionisation energy (IE) of DIP thin films $\left(\mathrm{IE}_{\mathrm{DIP}}=5.40 \mathrm{eV}\right),{ }^{33}$ which would in principle allow integer $\mathrm{CT}$ across their interface. However, we note that the formation of a CPX was observed in co-deposited films (i.e., mixed on a molecular scale) of DIP and F6. ${ }^{34,35}$ The frontier energy levels of DIP, F6, and their CPX [obtained from the present photoelectron spectroscopy (PES) data] are given in Fig. 1b. The influence of D-A junction formation on the electrical characteristics of OFETs was assessed by comparing DIP-based OFETs (Fig. 1c) before and after deposition of a F6 layer. After heterojunction formation we observe clearly improved hole transport, suggesting p-doping of DIP even in the bulk. Finally, with the help of electrostatic modelling, we can explain the hole enrichment throughout the DIP layer by $E_{\mathrm{F}}$-pinning induced CT across the D-A interface, resulting in an electrostatic potential drop over the entire film thickness. The latter moves $E_{\mathrm{F}}$ closer to the DIP HOMO level even at the interface to the far-away gate dielectric, i.e., remote p-doping takes place. Furthermore, we find evidence for CPX formation right at the DIP/F6 interface, which, however, significantly reduces hole accumulation in DIP, compared to a scenario without these complexes.

\section{Materials and methods}

DIP was purchased from Institut für PAH Forschung (Greifenberg, Germany) and F6 from Novaled (Dresden, Germany). Both materials were used without further purification, and deposited by thermal evaporation. The nominal film thickness was monitored by a quartz microbalance. For OFET measurements, highly p-doped silicon wafers with $300 \mathrm{~nm}$ oxide $\left(\mathrm{SiO}_{2}\right.$, dry oxidation) were covered with $20 \mathrm{~nm}$ of poly(methyl methacrylate) (PMMA) as oxide passivation layer, ${ }^{38}$ via spin coating from $1.0 \mathrm{wt} \%$ solution in xylene. After deposition of a $25 \mathrm{~nm}$ DIP film, nickel electrodes $(100 \mathrm{~nm})$ were deposited through a shadow mask. The channel width was $3 \mathrm{~mm}$ and the channel length $65 \mu \mathrm{m}$. Subsequently, a $5 \mathrm{~nm}$ thick F6 layer was deposited on top of the structure to form the D-A interface. PES and near edge X-ray absorption fine structure spectroscopy (NEXAFS) measurements were performed with highly p-doped silicon wafers with a native oxide layer $\left(\mathrm{SiO}_{x}, \approx 2 \mathrm{~nm}\right)$ as substrate, to avoid sample charging. The same doping type of the applied silicon wafers ensure similar work function and therefore similar electronic behavior. All substrates were solvent cleaned before use.

Electrical characterisation was done with a Keithley 4200SCS under vacuum conditions and using a transfer system in order to prevent devices from ambient exposure. The switch-on voltage $^{39}$ was determined by the onset of the displacement current, where charge carrier injection and formation of a conductive channel starts. ${ }^{40}$ The mobilities were estimated from the linear region of the transfer characteristics. ${ }^{41}$ The area-normalized capacitance $(C)$ of the whole gate insulator stack was determined via impedance measurements of capacitors using a Solartron Dielectric Interface 1296 and a Solartron Impedance/Gain-Phase Analyzer SI 1260.

PES and NEXAFS measurements were performed at the dipole beamline D1011 of the synchrotron storage ring MAX II at MAX IV laboratory (Lund University, Sweden). Ultraviolet photoelectron spectroscopy (UPS) to acquire the valence spectra 
was performed using a photon energy of $50 \mathrm{eV}$, and for measurements of the $\mathrm{C} 1 \mathrm{~s}$ core level region with X-ray photoelectron spectroscopy (XPS) we used $335 \mathrm{eV}$ excitation. Analyser pass energies of 20 and $50 \mathrm{eV}$ were chosen, respectively. With the SCIENTA SES200 electron energy analyser this gave an experimental resolution of $c a$. $50 \mathrm{meV}$ for the valence region, as determined from the width of the Fermi edge of a polycrystalline gold sample. The secondary electron cut-off (SECO) spectra were measured with a bias of $-26.84 \mathrm{~V}$ applied to the sample to overcome the analyser work function. X-ray absorption spectra were obtained by measuring the sample current (total electron yield). For energy calibration a pristine highly oriented pyrolytic graphite (HOPG) sample was used (absorption maximum of the C $1 \mathrm{~s}-\pi^{*}$ transition at $\left.285.40 \mathrm{eV}\right) .{ }^{42}$ All peak positions were determined by fitting Gaussians if not stated otherwise. Core level spectra were fitted using the software XPSPEAK 4.1 applying a combination of Shirley ${ }^{43,44}$ and linear background.

Optical absorption was measured using a PerkinElmer LAMBDA 950 UV-vis-NIR spectrophotometer in transmission mode using quartz glass substrates. Ex situ scanning force microscopy measurements were performed with a Bruker Dimension Icon in peak force tapping mode (ScanAsyst) and with a NanoWizard 3 AFM by JPK Instruments in standard tapping mode. Background correction and image analysis was done using the software package Gwyddion. ${ }^{45}$ Electrostatic modelling was performed according to ref. 14 and 46. The parameters used are given in the ESI. $\dagger$

\section{Results}

The transfer characteristics of a DIP-based OFET before and after F6 deposition in the linear regime are displayed in Fig. 2. Both measurements, i.e., without and with the presence of the D-A junction, reveal ambipolar behaviour, as notable sourcedrain current is detected for electron and hole accumulation, respectively. Bipolar transport in DIP was reported earlier, and

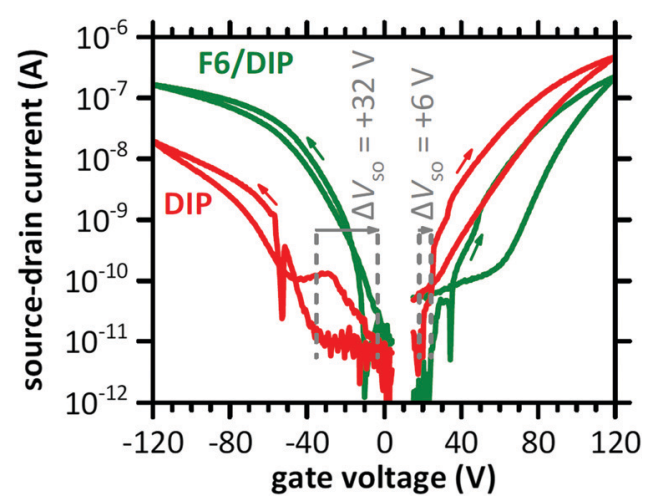

Fig. 2 Transfer characteristics of a DIP-based OFET before (DIP) and after deposition of F6 (F6/DIP). The dashed lines indicate the switch-on voltage $\left(V_{\mathrm{SO}}\right)$ and $\Delta V_{\mathrm{SO}}$. The applied drain voltage was $+2 \vee$ for the electrontransporting and $-2 \mathrm{~V}$ for the hole-transporting regime. The arrows mark the scan directions. The sudden drops in source-drain current, at $-53 \mathrm{~V}$ and $+35 \mathrm{~V}$, are due to the change of accumulated charge carrier type and thus of the sign of current (for the logarithmic scale absolute current values are used). For further details see Fig. S1 (ESI†). even higher electron mobility compared to hole mobility in single crystals ${ }^{27}$ and OFETs with a passivation layer between silicon oxide gate dielectric and the semiconductor. ${ }^{28,47}$ After deposition of F6, the curves at negative gate voltage (hole accumulation in DIP) are shifted to less negative voltage. This indicates improved hole accumulation in DIP, which is also expressed in a shift of the switch-on voltage $\left(\Delta V_{\text {SO }}\right)$ by $+32 \mathrm{~V}$. The curves for positive gate voltage (electron accumulation in DIP) are shifted to more positive voltage upon $\mathrm{D}-\mathrm{A}$ junction formation, and $\Delta V_{\text {SO }}$ is about $+6 \mathrm{~V}$. Apparently, electron accumulation in DIP is now less favourable. More details on $\Delta V_{\text {So }}$ determination via displacement currents are given in the ESI $\dagger$ (Fig. S1).

As the switch-on voltage is related to the density of charge carriers at the gate insulator, ${ }^{39,41,48}$ the change in density of accumulated charge carriers at the gate upon F6 deposition $(\Delta N)$ can be calculated by

$$
\Delta V_{\mathrm{SO}}=\frac{e \cdot \Delta N}{C}
$$

where, $e$ is the elementary charge and $C=10.09 \mathrm{nF} \mathrm{cm}{ }^{-2}$ is the area-normalized capacitance of the gate dielectric. With this we find $\Delta N=0.02 \mathrm{e} \mathrm{nm}^{-2}$, or 0.006 e per molecule (using a footprintarea of $0.3031 \mathrm{~nm}^{2}$ per DIP molecule in the crystal structure of the $\sigma$-phase $\left.\mathrm{e}^{33,49}\right)$. Somewhat higher values of $\Delta N$ were found for other D-A combinations, e.g. , ca. $0.04 \mathrm{e} \mathrm{nm}^{-2}$ for $\mathrm{Cu}$-phthalocyanine and its perfluorinated version ${ }^{10,11}$ and $c a$. $0.06 \mathrm{e} \mathrm{nm}^{-2}$ for pentacene and tetrafluoro-tetracyano-quinodimethane. ${ }^{8}$

Further evaluation of the transfer characteristics in the linear regime gives an estimate for the effective charge carrier mobility $\mu_{\mathrm{h}}$ (holes) and $\mu_{\mathrm{e}}$ (electrons), ${ }^{50,51}$ as summarized in Table 1. After deposition of F6, $\mu_{\mathrm{h}}$ increased $c a$. threefold while $\mu_{\mathrm{e}}$ decreased to $\mathrm{ca}$. half the value found for DIP alone. Furthermore, for the hole transport regime hysteresis is slightly reduced and in the electron transport regime slightly increased upon D-A junction formation. The changes of mobility and hysteresis are in line with enhanced hole accumulation as concluded on already above from changes of $V_{\text {so }}$. The higher hole density within DIP enhances the carrier density dependent mobility $^{52,53}$ and reduces the density of active hole traps. ${ }^{54,55}$ The opposite holds thus for the electron transport regime. Whereas electrons are the majority charge carriers in the DIP OFET before F6 deposition, the majority changes to holes after F6 deposition. Yet, in the light of the energy offset between IE and $\mathrm{EA}_{\mathrm{F} 6}$ (see Fig. 1b) that would support CT with high efficiency, it appears remarkable that from OFET characteristics we derive that only one additional out of $c a$. 170 DIP molecules at the interface to the gate dielectric becomes charged. To obtain further insight into this D-A interface, we performed additional experiments as described in the following.

Table 1 Electron $\left(\mu_{\mathrm{e}}\right)$ and hole $\left(\mu_{\mathrm{h}}\right)$ mobility in the linear transport regime as extracted from the transfer characteristics shown in Fig. 2

\begin{tabular}{lll}
\hline Channel & $\mu_{\mathrm{h}}\left(\mathrm{cm}^{2} \mathrm{~V}^{-1} \mathrm{~s}^{-1}\right)$ & $\mu_{\mathrm{e}}\left(\mathrm{cm}^{2} \mathrm{~V}^{-1} \mathrm{~s}^{-1}\right)$ \\
\hline DIP & $1 \times 10^{-3}$ & $1 \times 10^{-2}$ \\
F6/DIP & $3 \times 10^{-3}$ & $5 \times 10^{-3}$
\end{tabular}


For PES measurements we employ silicon wafers with a native surface oxide layer $\left(\mathrm{SiO}_{x}\right)$ as substrates to avoid sample charging that would occur for the $\mathrm{PMMA} / \mathrm{SiO}_{2} / \mathrm{Si}$ substrates (as used in OFETs). Thus, we first establish that the morphology and molecular orientation of the $\mathrm{D}-\mathrm{A}$ junction is comparable (a) DIP/PMMA

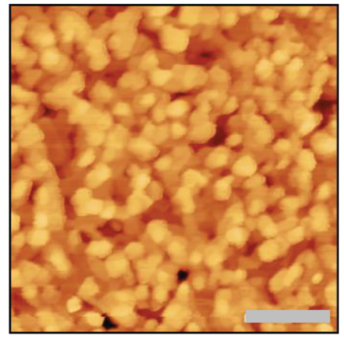

(c) F6/DIP/PMMA

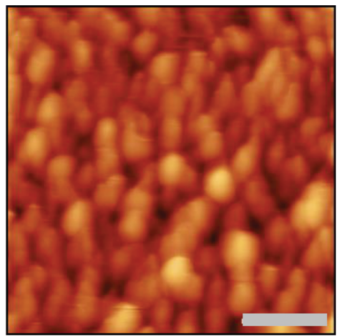

(b) $\mathrm{DIP} / \mathrm{SiO}_{\mathrm{x}}$

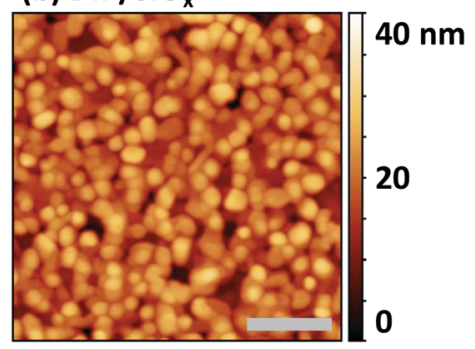

(d) F6/DIP/SiO

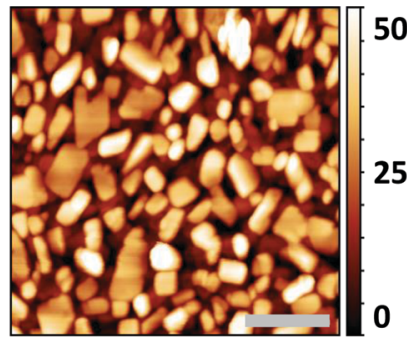

Fig. 3 Scanning force micrographs $\left(2 \times 2 \mu \mathrm{m}^{2}\right)$ for $25 \mathrm{~nm}$ DIP on PMMA/ $\mathrm{SiO}_{2} / \mathrm{Si}$ (a) and $30 \mathrm{~nm}$ DIP on $\mathrm{SiO}_{x}$ (b) as well as for $5 \mathrm{~nm} \mathrm{F6}$ on DIP/PMMA/ $\mathrm{SiO}_{2} / \mathrm{Si}$ (c) and $8.5 \mathrm{~nm} \mathrm{F6}$ on DIP/SiO $/$ / Si (d). The grey bars indicate a length of $500 \mathrm{~nm}$. on both types of substrates. Scanning force micrographs of DIP films deposited onto $\mathrm{PMMA} / \mathrm{SiO}_{2} / \mathrm{Si}$ and on $\mathrm{SiO}_{x} / \mathrm{Si}$ are shown in Fig. 3a and b. On both substrates, we observe the typical granular appearance of polycrystalline DIP films, with similar grain size of around $100 \mathrm{~nm}$, as previously reported for comparable films. ${ }^{56}$ For DIP on $\mathrm{PMMA} / \mathrm{SiO}_{2} / \mathrm{Si}$, we performed X-ray reflectivity measurements (see ESI, $\uparrow$ Fig. S2) and identify the presence of the $\sigma$-phase of DIP. ${ }^{33,49}$ Angle resolved NEXAFS was performed for DIP on $\mathrm{SiO}_{x} / \mathrm{Si}$ substrates and the $\pi$-system of the molecules is oriented at $83^{\circ}$ with respect to the surface, which is in accordance with the $\sigma$-phase of DIP, ${ }^{21}$ with molecules essentially upright standing on the substrate. Due to the similarity of the DIP film morphology found for the $\mathrm{SiO}_{x} / \mathrm{Si}$ substrate we suggest that the same structure and molecular orientation prevails here; furthermore, it is known that DIP molecules exhibit predominantly an upright standing orientation on silicon oxide surfaces. ${ }^{57}$ Scanning force micrographs of F6 deposited on top of DIP on both substrates are shown in Fig. $3 c$ and d. Once more we find a similar granular morphology for both samples, while the image for the D-A heterojunction on the polymer substrate appears more blurred due to non-ideal tip condition.

Since we find comparable morphological and structural properties of the DIP and F6/DIP films grown on the two substrates, we proceed with characterizing the electronic properties by PES. The corresponding valence region, the secondary electron cut-off (SECO), and the C 1s core levels spectra are shown in Fig. 4. The work function of the pristine $30 \mathrm{~nm}$ thick DIP film is $4.15 \mathrm{eV}$, equal to the work function we found for the bare $\mathrm{SiO}_{x} / \mathrm{Si}$ substrate (not shown), and thus vacuum level alignment occurs (a) SECO

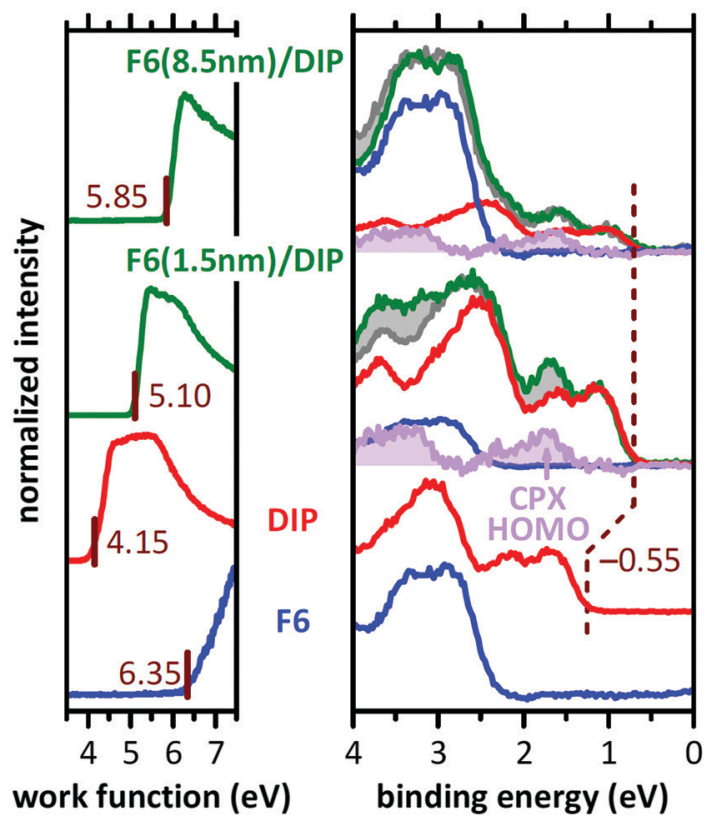

(c) C 1s core level

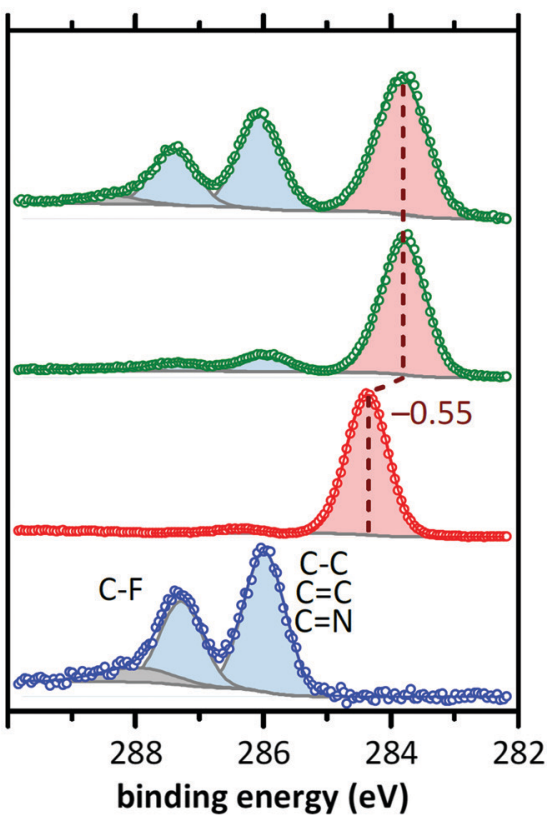

Fig. 4 Photoelectron spectroscopy data for pristine F6 and DIP films, as well as for F6 (1.5 nm and $8.5 \mathrm{~nm}$ ) deposited on the DIP film. (a) SECO spectra, (b) the valence region, and (c) C 1s core level region. After subtraction of the scaled DIP (red) and F6 (blue) spectra from the F6/DIP layered structure (green), the residual signal is attributed to the CPX (purple). The deconvolution of the C 1s spectra shows the contributions of DIP (light red), F6 (light blue) and shake-up satellites (grey). 
for the DIP/SiO $x$ interface. The HOMO level onset of DIP is $1.25 \mathrm{eV}$ below the Fermi level $\left(E_{\mathrm{F}}\right)$ and the resulting $\mathrm{IE}_{\mathrm{DIP}}$ is $5.40 \mathrm{eV}$, as also found in literature. ${ }^{33}$ As the transport gap of DIP was reported to be between 2.25 and $2.60 \mathrm{eV},^{36,58,59} E_{\mathrm{F}}$ is roughly mid-gap for the bare DIP film.

In contrast, the pristine F6 film exhibits a work function of $6.35 \mathrm{eV}$ and the HOMO onset is $c a .2 .35 \mathrm{eV}$ below $E_{\mathrm{F}}$, resulting in an $\mathrm{IE}_{\mathrm{F} 6}$ of $8.70 \mathrm{eV}$. Reported $\mathrm{IE}_{\mathrm{F} 6}$ values in literature vary significantly, between $7.55 \mathrm{eV}^{32}$ and $8.1 \mathrm{eV} .^{60}$ These variations in IE are most likely due to differences in the molecular orientation of F6 in the studied samples. ${ }^{59,61}$ Furthermore, recent theoretical work pointed out that the IE of F6 also shows a strong dependence on the molecular host environment, predicting $\mathrm{IE}_{\mathrm{F} 6}$ values as high as $8.82 \mathrm{eV} .^{62}$ However, the high sample work function found here and the high binding energy of the F6 HOMO level are a clear indication that the LUMO level of F6 is pinned at $E_{\mathrm{F} \cdot}{ }^{46,63}$

The $\mathrm{C}$ 1s core level spectrum of DIP exhibits a single peak, since all carbon atoms are in a very similar chemical environment. For a pure F6 film we find two well-separated peaks in the $\mathrm{C} 1 \mathrm{~s}$ region. The low binding energy component originates from carbons bound to other carbon or nitrogen atoms $(\mathrm{C}-\mathrm{C}, \mathrm{C}=\mathrm{C}$, $\mathrm{C} \equiv \mathrm{N}$ ), while the high binding energy component stems from carbon atoms bound to fluorine. ${ }^{64}$

To investigate the formation and electronic properties of the D-A interface, we deposited F6 of two different nominal thicknesses onto DIP. The thickness of $1.5 \mathrm{~nm}$ corresponds to $\mathrm{ca}$. a monolayer of F6 and should thus represent the very interface. The higher thickness of $8.5 \mathrm{~nm} \mathrm{F6}$ corresponds to multilayer of the acceptor, and is thus representative of a region farther from the interface. Furthermore, the strength of DIP feature attenuation by $\mathrm{F} 6$ allows inferring whether a comparably sharp or inter-diffused interface is formed (see discussion of Fig. 5 below). Upon F6 deposition onto DIP, the sample work function increases from $4.15 \mathrm{eV}$ (pristine DIP) to $5.10 \mathrm{eV}$ ( $1.5 \mathrm{~nm} \mathrm{~F} 6)$ and to $5.85 \mathrm{eV}$ (8.5 nm F6). The high work function is comparable to the reported $\mathrm{EA}_{\mathrm{F} 6}$ of $5.60 \mathrm{eV},{ }^{32}$ and thus the $\mathrm{F} 6$ multilayer is Fermi level pinned at the LUMO. ${ }^{17}$ This, in turn, implies that electrons have been transferred from DIP to F6 across the interface. The DIP HOMO level onset shifts by $0.55 \mathrm{eV}$ towards $E_{\mathrm{F}}$ upon F6 deposition,

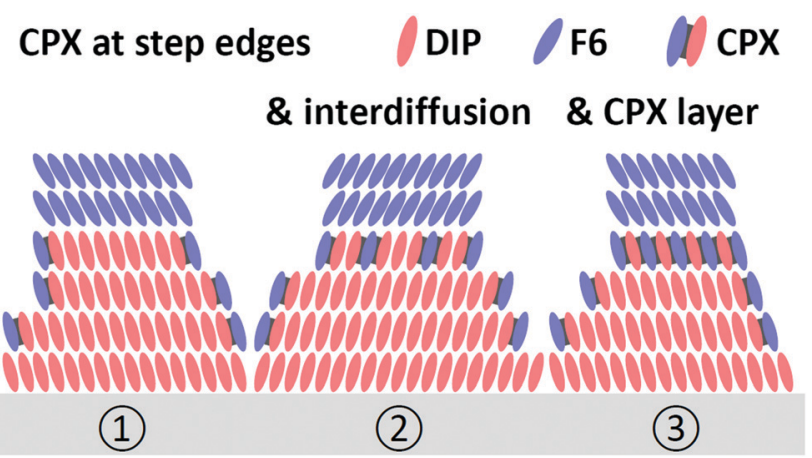

Fig. 5 Schemes of possible mechanisms for CPX formation at the corrugated surface of a DIP film in the presence of F6. (1) CPX formation at step edges. (2) additional inter-diffusion of DIP and F6 at the very interface, and (3) possible formation of a CPX interlayer. which brings it to a position close to or even at being $E_{\mathrm{F}}$-pinned. ${ }^{33}$ The signatures of DIP and F6 can be clearly identified in the C 1s core level spectrum of the respective samples (Fig. 4c). The binding energy shift of $0.55 \mathrm{eV}$ for the DIP C 1s level parallels that observed for its valence features, evidencing the electrostatic origin of the shift.

In a more detailed analysis, we scaled (in intensity) and shifted (in energy) the valence spectra of pristine DIP and F6 and subtracted these from the valence spectrum of $1.5 \mathrm{~nm}$ F6 on DIP. There is a clear residual signal (purple in Fig. 4b) with an onset at $0.7 \mathrm{eV}$ higher binding energy than that of DIP, and its shape does not resemble that of any of the pristine compounds. Therefore, we attribute this signal to the valence spectrum of a CPX formed by DIP and F6, right at the interface. Taking the three spectral signatures (DIP, F6, CPX) obtained in the just described manner, we can adequately reproduce the valence spectrum of $8.5 \mathrm{~nm} \mathrm{F6}$ on DIP (with appropriately scaled intensity). Note that the intensity of the CPX signature is lower for $8.5 \mathrm{~nm}$ F6 coverage than for $1.5 \mathrm{~nm}$ F6 coverage, supporting the notion that the CPX is confined to the very interface. Any signs of CPX formation are not seen in the C 1s core level spectra, as only the frontier orbitals are involved in complex formation. ${ }^{7,65}$ Considering a symmetric gap opening upon CPX formation ${ }^{7}$ between DIP and F6, the IE of the CPX is $0.7 \mathrm{eV}$ higher that the IE of DIP (see Fig. 4b), and the EA of the $\mathrm{CPX}$ is $0.7 \mathrm{eV}$ lower than the $\mathrm{EA}$ of $\mathrm{F} 6$, i.e., we obtain $\mathrm{IE}_{\mathrm{CPX}}=$ $6.1 \mathrm{eV}$ and $\mathrm{EA}_{\mathrm{CPX}}=4.9 \mathrm{eV}$, as shown in Fig. 1b. In contrast to the $E_{\mathrm{F}}$-pinned DIP (at the HOMO) and F6 (at the LUMO) on either side of the junction, the interfacial CPXs appear unpinned. The presence of the CPXs thus adds additional complexity to the electronic structure of the $\mathrm{D}-\mathrm{A}$ heterojunction.

To provide further support for the presence of CPXs at the F6/DIP interface, optical absorption measurements were performed on a multilayer stack with $10 \mathrm{D}-\mathrm{A}$ interfaces to increase the fraction of interfaces in the sample. We find the same lowenergy absorption features (at $c a$. 1.0 and $1.4 \mathrm{eV}$ ) that were reported for CPXs of DIP and F6 in co-deposited films where mixing occurred on a molecular scale. ${ }^{34}$ Furthermore, a CPXrelated absorption feature was also detected in X-ray absorption measurements on a two-layer junction. Optical and X-ray absorption data are shown in the ESI $\dagger$ (Fig. S3 and S4). The analysis of molecular orientation from X-ray absorption in the $\mathrm{ESI} \dagger$ further shows that the deposition of F6 does not induce a re-orientation of the upright standing DIP molecules.

Since CPX formation requires the overlap of the donor HOMO and acceptor LUMO, both of which are delocalized orbitals in the plane of the molecules, the following morphological considerations are required. When F6 molecules adsorb on the surface of a DIP film with upright standing molecules, orbital overlap and thus CPX formation are precluded. Therefore, mechanisms enabling such orbital overlap must be present. One obvious possibility is, given the granular appearance and relatively high corrugation of the DIP films (Fig. 3), that F6 molecules attach at the edges of DIP grains and form CPXs via the sideways overlap of the two $\pi$-electron systems. However, it is unlikely that this is the only CPX enabling mechanism as this would lead 
to a comparably small fraction of interfacial CPXs only. Fig. 5 shows schematic structures for the appearance of the CPX at the F6/DIP interface. In addition, inter-diffusion of F6 and DIP would also enable orbital overlap and CPX formation. Such a CPX-containing interlayer is most probably confined to one or a few molecular layers near the interface, as our photoemission data (Fig. 4b) reveal a strong attenuation of CPX features by few $\mathrm{nm}$ further F6 deposition. Given the high surface sensitivity of photoemission ( $c a .1 \mathrm{~nm}$ with our experimental parameters), more vertically pronounced intermixing and CPX formation would results in stronger persistence of the diagnostic CPX signal for higher F6 thickness.

Finally, electrostatic modelling of the investigated structures helps to obtain insight into the charge density re-distribution upon D-A junction formation and the electrostatic potential landscape across the multilayer structures, based on experimental parameters. We note that in the 1-dimensional models used here interface corrugation cannot be included, which limits the quantitative significance of the models. Qualitative insight, however, is fully provided and summarized in Fig. 6. Four different structures were investigated: without and with a gate dielectric (insulator) between conductive substrate (degenerately doped $\mathrm{Si}$ ) and the organic layers to mimic the experimental situation in UPS and OFETs, respectively, and each without and with a monolayer CPX at the interface, to unravel its impact on interfacial charge density. The material parameters used for the models are given in the ESI $\dagger$ (Table S1).

First of all, vacuum level alignment holds for all interfaces between DIP film the substrates, since the HOMO and LUMO levels of the organic semiconductor are far away from $E_{\mathrm{F}}$, and flat energy levels prevail in the DIP layer before adding the acceptor layer. This fully agrees with the finding from PES experiments described above. The addition of an F6 layer on top of the four structures then induces significant charge density rearrangements, most notable at the D-A junction. As seen in Fig. 6a, where we excluded the interfacial CPX, the LUMO of F6 is $E_{\mathrm{F}}$-pinned due to electron transfer from DIP, whose HOMO becomes accordingly $E_{\mathrm{F}}$-pinned at the interface. The charges induced on both sides of the interface (shown in the bottom row of Fig. 6) result in space charge layers and the corresponding energy level bending on either side of the junction, ${ }^{66}$ more clearly seen for the F6 layer. The reason for this is the additional electrostatic potential drop across the entire DIP layer, which is a consequence of the fact that $E_{\mathrm{F}}$ is about mid-gap at the bottom interface to $\mathrm{Si}$ and it is pinned at the HOMO at the interface with F6. Next, we insert a monolayer of the CPX with its unpinned energy levels at the D-A interface, as shown in Fig. 6b. Charge transfer between DIP and F6 still takes place, as well as the accompanying $E_{\mathrm{F}}$-pinning, energy level bending and electrostatic potential drop across DIP. However, as seen in the bottom part of Fig. $6 \mathrm{~b}$, the magnitude of induced charges on either side of the junction is reduced considerably. In essence, the CPX layer acts as a thin insulating interlayer between $\mathrm{D}$ and $\mathrm{A}$, which reduces the amount of charge transferred across the interface. In both cases discussed so far, there is virtually no change in charge density at the bottom interface between DIP and the conductive Si.

The latter situation changes notably upon inserting a thick insulating layer (gate dielectric) between the conductive Si and DIP, as shown in Fig. $6 \mathrm{c}$ and d, mimicking the situation in an OFET.

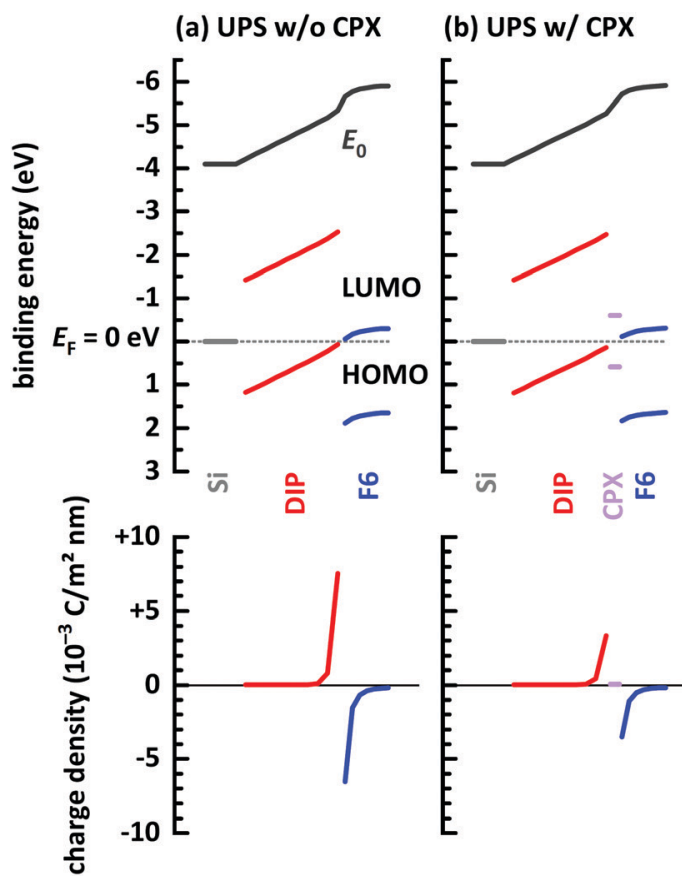

(c) OFET w/o CPX
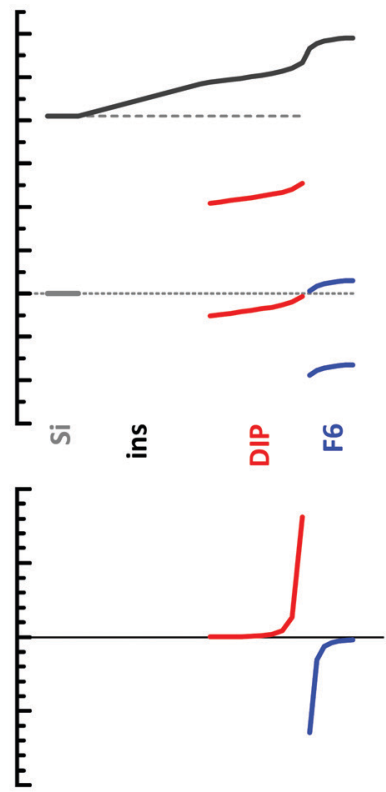

(d) OFET w/ CPX

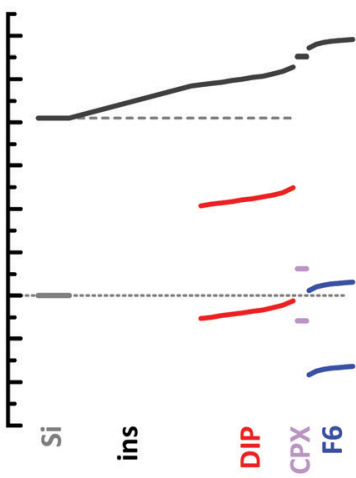

E

Fig. 6 Energy level diagrams obtained from electrostatic modeling, ${ }^{14,46}$ after reaching electronic equilibrium. (a) Conductive Si/10 layers DIP/7 layers F6 molecules. (b) Same as (a) but with a monolayer CPX between DIP and F6. (c) Conductive Si/20 nm insulator (ins)/10 layers DIP/7 layers F6 molecules. (d) Same as (c) but with a monolayer CPX between DIP and F6. Input parameters for the models are given in ESI. $\dagger$ 
The insulating layer has a wide energy gap and thus Fermi level pinning is absent for this material. Vacuum level alignment and flat energy level are present in the stack including DIP before addition of the acceptor layer. Upon addition of F6, charge density re-arrangement happens in a mostly analogous manner as described above for Fig. 6a and b. As well, the HOMO and LUMO levels of DIP and F6, respectively, become $E_{\mathrm{F}}$-pinned, energy level bending away from the interface is observed, and an electrostatic potential drop across DIP occurs. However, there is a notable change in the spatial electrostatic potential drop distribution that the gate dielectric insulator is present. Now, the Fermi level is determined on the left-hand side of the structure by the conductive Si contacting the insulator, and on the right-and side by the $E_{\mathrm{F}}$-pinned HOMO level of DIP. Consequently, the electrostatic potential difference between these two positions leads to a potential drop across both the insulator layer and the DIP layer. In turn, this moves the DIP occupied levels closer to $E_{\mathrm{F}}$ at any position in the layer, i.e., including the region close to the insulator, as clearly seen in Fig. $6 \mathrm{c}$ and $\mathrm{d}$. This is the main reason for the observed increase of accumulated holes $\Delta N$ in the OFET (see Fig. 2 and its discussion). Finally, we also observe, in the bottom row plots of Fig. $6 \mathrm{c}$ and d, for this case that the insertion of a monolayer CPX between DIP and F6 significantly reduces the induced carrier density on either side of the D-A interface. When aiming at a high density of interface-doping induced charge carriers, the formation of CPXs should thus be prohibited.

\section{Summary}

We unravelled the mechanism that leads to increased hole density in the organic semiconductor DIP, next to a gate dielectric in an OFET structure, upon deposition of a molecular acceptor layer on top. Electron transfer from DIP to the acceptor F6 readily occurs across this D-A interface because $\mathrm{IE}_{\mathrm{DIP}}$ is lower than $\mathrm{EA}_{\mathrm{F6}}$. Due to this interfacial integer charge transfer, the HOMO of DIP and LUMO of F6 become $E_{\mathrm{F}}$-pinned at the junction, and energy level bending away from the interface on both sides occurs. In addition, also an interlayer of CPXs containing DIP and F6 molecules is formed, which corresponds to a thin insulator. This, in turn, reduces the density of integer charge transfer across the D-A interface compared to the situation without CPXs, as found from electrostatic modelling. Furthermore, in OFETs the presence of the gate dielectric enhances the acceptor-induced hole density at the DIP/dielectric interface. This is the result of a distributed electrostatic potential drop across the dielectric and the semiconductor layer, which moves the HOMO levels of DIP closer to $E_{\mathrm{F}}$ compared to a structure without the dielectric. Our results highlight the importance of the gate dielectric for understanding the spatial carrier density distribution in OFETs that employ D-A interfaces for enhancing the performance. For such structures, however, the formation of CPXs at the interface should be inhibited, as this reduces the beneficial effect of increased carrier density via interfacial charge transfer. A possible acceptor to avoid CPX formation with DIP could be the non-planar molybdenum tris[1,2-bis-(trifluoromethyl)ethane-1,2-dithiolene $]\left[\mathrm{Mo}(\mathrm{tfd})_{3}\right]$. Phase separation and the absence of complex formation was reported for co-deposited films of DIP and $\mathrm{Mo}(\mathrm{tfd})_{3} \cdot{ }^{67}$ Finally, we note that similar considerations may be relevant for multilayer OFET channels, and the type of electrostatic modeling employed here can provide valuable insight into the charge distribution in such devices, which, e.g., can achieve superior carrier mobility. ${ }^{68}$

\section{Conflicts of interest}

There are no conflicts to declare.

\section{Acknowledgements}

This work was supported by the Deutsche Forschungsgemeinschaft (DFG) - project 239543752. We are grateful for beamtime at beamline D1011 at Max IV laboratory (Lund, Sweden). We thank Justas Jasiunas for OFET and X-ray reflectivity measurements performed during his internship at the University of Augsburg.

\section{References}

1 C. W. Tang, Appl. Phys. Lett., 1986, 48, 183-185.

2 K. Goushi, K. Yoshida, K. Sato and C. Adachi, Nat. Photonics, 2012, 6, 253-258.

3 K. Walzer, B. Männig, M. Pfeiffer, K. Leo, B. Maennig, M. Pfeiffer and K. Leo, Highly efficient organic devices based on electrically doped transport layers, American Chemical Society, 2007, vol. 107.

4 I. Salzmann, G. Heimel, M. Oehzelt, S. Winkler and N. Koch, Acc. Chem. Res., 2016, 49, 370-378.

5 R. Sato, M. Dogishi, T. Higashino, T. Kadoya, T. Kawamoto and T. Mori, J. Phys. Chem. C, 2017, 121, 6561-6568.

6 B. Siegmund, A. Mischok, J. Benduhn, O. Zeika, S. Ullbrich, F. Nehm, M. Böhm, D. Spoltore, H. Fröb, C. Körner, K. Leo and K. Vandewal, Nat. Commun., 2017, 8, 15421.

7 H. Méndez, G. Heimel, A. Opitz, K. Sauer, P. Barkowski, M. Oehzelt, J. Soeda, T. Okamoto, J. Takeya, J.-B. Arlin, J.-Y. Balandier, Y. Geerts, N. Koch and I. Salzmann, Angew. Chem., Int. Ed., 2013, 52, 7751-7755.

8 Y. Abe, T. Hasegawa, Y. Takahashi, T. Yamada and Y. Tokura, Appl. Phys. Lett., 2005, 87, 1-3.

9 C. Videlot-Ackermann, J. Ackermann and F. Fages, Synth. Met., 2007, 157, 551-557.

10 H. Wang, J. Wang, H. Huang, X. Yan and D. Yan, Org. Electron., 2006, 7, 369-374.

11 K. Eguchi, M. M. Matsushita and K. Awaga, J. Phys. Chem. C, 2018, 122, 26054-26060.

12 F. Hong, F. Xing, W. Gu, W. Jin, J. Zhang and J. Wang, Synth. Met., 2010, 160, 475-478.

13 H. Wang and D. Yan, NPG Asia Mater., 2010, 2, 69-78.

14 M. Oehzelt, K. Akaike, N. Koch and G. Heimel, Sci. Adv., 2015, 1, e1501127.

15 H. Y. Mao, F. Bussolotti, D. C. Qi, R. Wang, S. Kera, N. Ueno, A. T. S. Wee and W. Chen, Org. Electron., 2011, 12, 534-540. 
16 P. W. Chye, I. A. Babalola, T. Sukegawa and W. E. Spicer, Phys. Rev. Lett., 1975, 35, 1602-1604.

17 J.-P. P. Yang, L.-T. T. Shang, F. Bussolotti, L.-W. W. Cheng, W.-Q. Q. Wang, X.-H. H. Zeng, S. Kera, Y.-Q. Q. Li, J.-X. X. Tang and N. Ueno, Org. Electron., 2017, 48, 172-178.

18 H. Alves, A. S. Molinari, H. Xie and A. F. Morpurgo, Nat. Mater., 2008, 7, 574-580.

19 K. Xu, H. Sun, T. P. Ruoko, G. Wang, R. Kroon, N. B. Kolhe, Y. Puttisong, X. Liu, D. Fazzi, K. Shibata, C. Y. Yang, N. Sun, G. Persson, A. B. Yankovich, E. Olsson, H. Yoshida, W. M. Chen, M. Fahlman, M. Kemerink, S. A. Jenekhe, C. Müller, M. Berggren and S. Fabiano, Nat. Mater., 2020, 1-7.

20 A. Opitz, J. Phys.: Condens. Matter, 2017, 29, 133001.

21 U. Hörmann, C. Lorch, A. Hinderhofer, A. Gerlach, M. Gruber, J. Kraus, B. Sykora, S. Grob, T. Linderl, A. Wilke, A. Opitz, R. Hansson, A. S. Anselmo, Y. Ozawa, Y. Nakayama, H. Ishii, N. Koch, E. Moons, F. Schreiber, W. Brütting and W. Brütting, J. Phys. Chem. C, 2014, 118, 26462-26470.

22 A. Opitz, A. Wilke, P. Amsalem, M. Oehzelt, R. P. Blum, J. P. Rabe, T. Mizokuro, U. Hormann, R. Hansson, E. Moons and N. Koch, Sci. Rep., 2016, 6, 21291.

23 K. Akaike, N. Koch, G. Heimel and M. Oehzelt, Adv. Mater. Interfaces, 2015, 2, 1500232.

24 N. Sai, R. Gearba, A. Dolocan, J. R. Tritsch, W. L. Chan, J. R. Chelikowsky, K. Leung and X. Zhu, J. Phys. Chem. Lett., 2012, 3, 2173-2177.

25 K. Vandewal, Annu. Rev. Phys. Chem., 2016, 67, 113-133.

26 M. Aghamohammadi, A. Fernández, M. Schmidt, A. PérezRodríguez, A. R. Goñi, J. Fraxedas, G. Sauthier, M. Paradinas, C. Ocal and E. Barrena, J. Phys. Chem. C, 2014, 118, 1483314839.

27 A. K. Tripathi and J. Pflaum, Appl. Phys. Lett., 2006, 89, 082103.

28 M. Horlet, M. Kraus, W. Brütting and A. Opitz, Appl. Phys. Lett., 2011, 98, 233304.

29 P. K. Koech, A. B. Padmaperuma, L. Wang, J. S. Swensen, E. Polikarpov, J. T. Darsell, J. E. Rainbolt and D. J. Gaspar, Chem. Mater., 2010, 22, 3926-3932.

30 Y. Karpov, T. Erdmann, M. Stamm, U. Lappan, O. Guskova, M. Malanin, I. Raguzin, T. Beryozkina, V. Bakulev, F. Günther, S. Gemming, G. Seifert, M. Hambsch, S. Mannsfeld, B. Voit and A. Kiriy, Macromolecules, 2017, 50, 914-926.

31 B. Lüssem, M. L. Tietze, H. Kleemann, C. Hoßbach, J. W. Bartha, A. Zakhidov and K. Leo, Nat. Commun., 2013, 4, 2775.

32 F. Zhang and A. Kahn, Adv. Funct. Mater., 2018, 28, 1703780.

33 J. Wagner, M. Gruber, A. Hinderhofer, A. Wilke, B. Bröker, J. Frisch, P. Amsalem, A. Vollmer, A. Opitz, N. Koch, F. Schreiber and W. Brütting, Adv. Funct. Mater., 2010, 20, 4295-4303.

34 G. Duva, L. Pithan, C. Zeiser, B. Reisz, J. Dieterle, B. Hofferberth, P. Beyer, L. Bogula, A. Opitz, S. Kowarik, A. Hinderhofer, A. Gerlach and F. Schreiber, J. Phys. Chem. C, 2018, 122, 18705-18714.

35 G. Duva, P. Beyer, R. Scholz, V. Belova, A. Opitz, A. Hinderhofer, A. Gerlach and F. Schreiber, Phys. Chem. Chem. Phys., 2019, 21, 17190-17199.
36 A. Wilke, J. Endres, U. Hörmann, J. Niederhausen, R. Schlesinger, J. Frisch, P. Amsalem, J. Wagner, M. Gruber, A. Opitz, A. Vollmer, W. Brütting, A. Kahn and N. Koch, Appl. Phys. Lett., 2012, 101, 233301.

37 H. Méndez, G. Heimel, S. Winkler, J. Frisch, A. Opitz, K. Sauer, B. Wegner, M. Oehzelt, C. Röthel, S. Duhm, D. Többens, N. Koch and I. Salzmann, Nat. Commun., $2015,6,8560$.

38 N. Benson, C. Melzer, R. Schmechel and H. von Seggern, Phys. Status Solidi, 2008, 205, 475-487.

39 E. J. Meijer, C. Detcheverry, P. J. Baesjou, E. Van Veenendaal, D. M. De Leeuw and T. M. Klapwijk, J. Appl. Phys., 2003, 93, 4831-4835.

40 Y. Liang, Dissertation, 2011.

41 S. M. Sze and D. C. Mattis, Phys. Today, 1970, 23, 75.

42 P. E. Batson, Phys. Rev. B: Condens. Matter Mater. Phys., 1993, 48, 2608-2610.

43 D. A. Shirley, Phys. Rev. B: Solid State, 1972, 5, 4709-4714. 44 J. Végh, J. Electron Spectrosc. Relat. Phenom., 2006, 151, 159-164. 45 D. Nečas and P. Klapetek, Cent. Eur. J. Phys., 2012, 10, 181-188. 46 M. Oehzelt, N. Koch and G. Heimel, Nat. Commun., 2014, $5,4174$.

47 A. Opitz, M. Horlet, M. Kiwull, J. Wagner, M. Kraus and W. Brütting, Org. Electron., 2012, 13, 1614-1622.

48 E. J. Meijer, C. Tanase, P. W. M. Blom, E. Van Veenendaal, B. H. Huisman, D. M. De Leeuw and T. M. Klapwijk, Appl. Phys. Lett., 2002, 80, 3838-3840.

49 A. C. Dürr, N. Koch, M. Kelsch, A. Rühm, J. Ghijsen, R. L. Johnson, J.-J. Pireaux, J. Schwartz, F. Schreiber, H. Dosch and A. Kahn, Phys. Rev. B: Condens. Matter Mater. Phys., 2003, 68, 115428.

50 T. Uemura, C. Rolin, T. H. Ke, P. Fesenko, J. Genoe, P. Heremans and J. Takeya, Adv. Mater., 2016, 28, 151-155.

51 Y. Xu, Y. Li, S. Li, F. Balestra, G. Ghibaudo, W. Li, Y. F. Lin, H. Sun, J. Wan, X. Wang, Y. Guo, Y. Shi and Y. Y. Noh, Adv. Funct. Mater., 2020, 30, 1904508.

52 W. F. Pasveer, J. Cottaar, C. Tanase, R. Coehoorn, P. A. Bobbert, P. W. M. Blom, M. De Leeuw and M. A. J. Michels, Phys. Rev. Lett., 2005, 94, 206601.

53 R. Coehoorn, W. F. Pasveer, P. A. Bobbert and M. A. J. Michels, Phys. Rev. B: Condens. Matter Mater. Phys., 2005, 72, 155206.

54 Y. Zhang, B. De Boer and P. W. M. Blom, Phys. Rev. B: Condens. Matter Mater. Phys., 2010, 81, 085201.

55 S. Olthof, S. Mehraeen, S. K. Mohapatra, S. Barlow, V. Coropceanu, J. L. Brédas, S. R. Marder and A. Kahn, Phys. Rev. Lett., 2012, 109, 176601.

56 A. C. Dürr, B. Nickel, V. Sharma, U. Täffner and H. Dosch, Thin Solid Films, 2006, 503, 127-132.

57 S. Kowarik, A. Gerlach, S. Sellner, F. Schreiber, L. Cavalcanti and O. Konovalov, Phys. Rev. Lett., 2006, 96, 125504.

58 S. Krause, A. Schöll and E. Umbach, Org. Electron., 2013, 14, 584-590.

59 W. N. Han, K. Yonezawa, R. Makino, K. Kato, A. Hinderhofer, R. Murdey, R. Shiraishi, H. Yoshida, N. Sato, N. Ueno and S. Kera, Appl. Phys. Lett., 2013, 103, 253301. 
60 M. Hantusch, R. Kuhrt and M. Knupfer, J. Phys. Chem. C, 2020, 124, 2961-2967.

61 S. Duhm, G. Heimel, I. Salzmann, H. Glowatzki, R. L. Johnson, A. Vollmer, J. P. Rabe and N. Koch, Nat. Mater., 2008, 7, 326-332.

62 J. Li, I. Duchemin, O. M. Roscioni, P. Friederich, M. Anderson, E. Da Como, G. Kociok-Köhn, W. Wenzel, C. Zannoni, D. Beljonne, X. Blase and G. D'Avino, Mater. Horiz., 2019, 6, 107-114.

63 H. Wang, P. Amsalem, G. Heimel, I. Salzmann, N. Koch and M. Oehzelt, Adv. Mater., 2014, 26, 925-930.

64 C. Christodoulou, A. Giannakopoulos, G. Ligorio, M. Oehzelt, M. Timpel, J. Niederhausen, L. Pasquali, A. Giglia, K. Parvez,
K. Müllen, D. Beljonne, N. Koch and M. V. Nardi, ACS Appl. Mater. Interfaces, 2015, 7, 19134-19144.

65 I. Salzmann, G. Heimel, S. Duhm, M. Oehzelt, P. Pingel, B. M. George, A. Schnegg, K. Lips, R.-P. P. Blum, A. Vollmer and N. Koch, Phys. Rev. Lett., 2012, 108, 035502.

66 J. X. Tang, C. S. Lee and S. T. Lee, J. Appl. Phys., 2007, 101, 064504.

67 A. Opitz, C. Peter, B. Wegner, B. Wegner, H. S. S. S. S. R. Matte, A. Röttger, T. Florian, X. Xu, P. Beyer, L. Grubert, S. Hecht, V. Belova, A. Hinderhofer, F. Schreiber, C. Kasper, J. Pflaum, Y. Zhang, S. Barlow, S. R. Marder, N. Koch and N. Koch, J. Phys. Chem. C, 2020, 124, 11023-11031.

68 P. Zhang, H. Wang and D. Yan, Adv. Mater., 2017, 29, 1702427. 